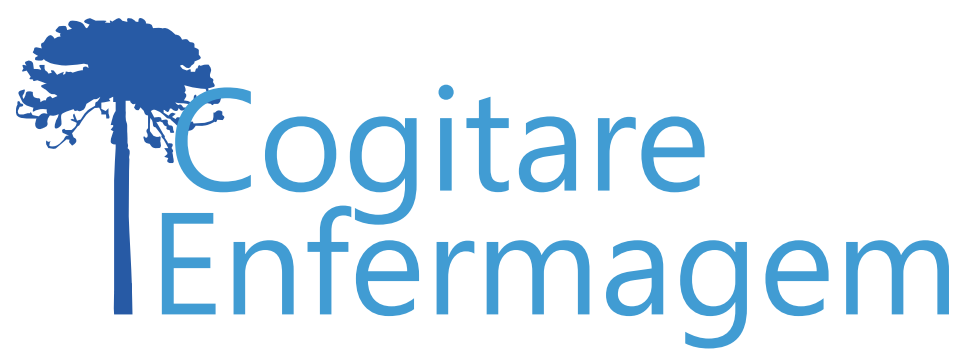

\title{
CUSTO-EFETIVIDADE DA CENTRAL DE MISTURA DE MEDICAMENTOS INJETÁVEIS EM UNIDADE DE TERAPIA INTENSIVA NEONATAL*
}

Lucília Feliciano Marques ${ }^{1}$, Cristiano Bertolossi Marta ${ }^{2}$, Roberto Carlos Lyra da Silva ${ }^{3}$, Antônio Augusto de Freitas Peregrino ${ }^{4}$, Carlos Roberto Lyra da Silva ${ }^{5}$, Vivian Schutz ${ }^{6}$

\section{RESUMO}

Objetivo: analisar o custo-efetividade da central de mistura intravenosa na redução de erros de doses no preparo de medicamentos injetáveis em terapia intensiva neonatal.

Método: modelo matemático proposto em 2017 para avaliar o custo-efetividade, comparando duas tecnologias em saúde em cenários hipotéticos utilizando o programa Software TreeAge® Pro Suite 2011. Simulação de Monte Carlo analisou a robustez do modelo.

Resultados: o modelo prediz que a central de mistura de injetáveis é custo-efetivo, com efetividade média de 0,96 para se evitar erros de dose na administração de medicamentos intravenosos, com razão de custo-efetividade incremental de $\mathrm{R} \$ 26.785,61$.

Conclusão: o uso da central de mistura intravenosa foi a alternativa mais custo-efetiva, considerando a disposição de pagar assumida no modelo. O estudo pode contribuir para reduzir incertezas na tomada de decisões acerca da incorporação de tecnologias para redução de erros de dose na administração de medicamentos em UTI pediátrica e neonatal.

DESCRITORES: Erros de medicação; Segurança do paciente; Análise de custo-efetividade; Unidades de terapia intensiva neonatal; Avaliação da tecnologia biomédica.

\footnotetext{
*Artigo extraído da tese de doutorado "Custo-efetividade da utilização da central de mistura intraveno-sa em unidade de terapia intensiva neonatal". Universidade Federal do Estado do Rio de Janeiro, 2017.
}

COMO REFERENCIAR ESTE ARTIGO:

Marques LF, Marta CB, Silva RCL da, Peregrino AA de F, Silva CRL da, Schutz V. Custo-efetividade da central de mistura de medicamentos injetáveis em unidade de terapia intensiva neonatal. Cogitare enferm. [Internet]. 2019 [acesso em "colocar data de acesso, dia, mês abreviado e ano"]; 24. Disponível em: http://dx.doi.org/10.5380/ ce.v24i0.60877.

Este obra está licenciado com uma Licença Creative Commons Atribuição 4.0 Internacional.

${ }^{1}$ Enfermeira. Doutora em Enfermagem. Enfermeira da Unidade de Terapia Neonatal do Bürgerhospital. Frankfurt, Alemanha. $(2$

${ }^{2}$ Enfermeiro. Doutor em Enfermagem. Docente de Enfermagem da Universidade do Estado do Rio de Janeiro. Rio de Janeiro, RJ, Brasil.

${ }^{3}$ Enfermeiro. Doutor em Enfermagem. Docente de Enfermagem da Universidade Federal do Estado do Rio de Janeiro. Rio de Janeiro, RJ, Brasil. (

${ }^{4}$ Enfermeiro. Doutor em Saúde Coletiva. Subcoordenador do Laboratório de Ciências Radiológicas Universidade do Estado do Rio de Janeiro. Rio de Janeiro, RJ, Brasil. 0

${ }^{5}$ Enfermeiro. Doutor em Enfermagem. Docente de Enfermagem da Universidade Federal do Estado do Rio de janeiro. Rio de Janeiro, RJ, Brasil.

${ }^{6}$ Enfermeira. Doutora em Enfermagem. Docente de Enfermagem da Universidade Federal do Estado do Rio de Janei-ro. Rio de Janeiro, RJ, Brasil. 


\title{
COST-EFFECTIVENESS OF THE INTRAVENOUS MEDICATION MIXING CENTER IN A NEONATAL INTENSIVE CARE UNIT
}

\begin{abstract}
Objective: To analyze the cost-effectiveness of the intravenous medication mixing center in reducing dose errors in the preparation of injectable medications in neonatal intensive care. Method: mathematical model proposed in 2017 to assess cost-effectiveness by comparing two health technologies in hypothetical scenarios using the TreeAge ${ }^{\circledR} 2011$ Pro Suite Software. A Monte Carlo simulation was used to analyze the robustness of the model.

Results: the model predicted that the intravenous medication mixing center was cost-effective, with a mean effectiveness of 0.96 in avoiding dose errors in intravenous drug administration, with an incremental cost-effectiveness ratio of $R \$ 26,785.61$.

Conclusion: the use of an intravenous medication mixing center was the most cost-effective alternative, considering the willingness to pay assumed in the model. The study may contribute to reducing uncertainty in decision making regarding the incorporation of dose error reduction technologies in medication administration in pediatric and neonatal ICUs.
\end{abstract}

DESCRIPTORS: Medication errors; Patient safety; Cost-effectiveness analysis; Neonatal intensive care units; Evaluation of biomedical technology.

\section{COSTO EFECTIVIDAD DE LA CENTRAL DE MEZCLA DE PRODUCTOS MEDICINALES INYECTABLES EN UNA UNIDAD DE TERAPIA INTENSIVA NEONATAL}

\begin{abstract}
RESUMEN:
Objetivo: analizar el costo efectividad de la central de mezcla intravenosa en la reducción de errores de dosis, en la preparación de medicamentos inyectables, en terapia intensiva neonatal.

Método: modelo matemático, propuesto en 2017, para evaluar el costo efectividad comparando dos tecnologías de la salud, en escenarios hipotéticos utilizando el programa Software TreeAge ${ }^{\circledR}$ Pro Suite 2011. La Simulación de Monte Carlo analizó el grado de robustez del modelo.

Resultados: el modelo predice que la central de mezclas de inyectables es costo efectivo para evitar errores de dosis en la administración de medicamentos intravenosos; la efectividad media fue de 0,96 y la razón de costo efectividad incremental de $R \$ 26.785,61$.

Conclusión: el uso de la central de mezcla intravenosa fue la mejor alternativa costo efectiva, considerando la disposición de pagar asumida en el modelo. El estudio puede contribuir para reducir incertidumbres en la toma de decisiones acerca de la incorporación de tecnologías para reducción de errores de dosis, en la administración de medicamentos en UTI pediátrica y neonatal.
\end{abstract}

DESCRIPTORES: Errores de medicación; Seguridad del paciente; Análisis de costo efectividad; Unidades de terapia intensiva neonatal; Evaluación da tecnología biomédica. 
O processo de medicação pode ser definido como um sistema complexo, em que erros podem ocorrer, acarretando injúrias ao paciente e gastos evitáveis. O neonato maior é mais suscetível devido a vários fatores e peculiaridades do recém-nascido (RN), como é o caso do cálculo da dose baseada na idade, peso e na imaturidade fisiológica, que altera a capacidade de absorção e excreção das drogas ${ }^{(1)}$.

Possivelmente $66 \%$ dos erros que ocorrem na Unidade de Terapia Neonatal (UTIN) são relacionados a medicamentos, e $38 \%$ são erros de dosagem ${ }^{(2)}$. Durante algumas etapas do processo de preparo e administração, erros na administração (72-75\%), documentação (17-21\%), dispensação (5-58\%) e prescrição (3-37\%) foram documentados ${ }^{(3)}$.

O Sistema de Distribuição de Medicamentos por Dose Unitária (SDMDU) tem possibilitado o acompanhamento farmacoterapêutico do usuário, além de propiciar uma distribuição do medicamento com segurança e rastreabilidade. Os medicamentos são distribuídos na forma pronta para uso, de acordo com a prescrição e sem necessidade de manipulação ${ }^{(4-7)}$.

Um avanço no serviço de Farmácia Hospitalar é a Central de Mistura Intravenosa, que pode garantir o uso racional do medicamento, que são manipulados e dispensados individualmente por farmacêuticos, reduzindo ocorrência de erros e melhorando a segurança na infusão(8).

Conforme RDC Anvisa n 67/2007, a manipulação de fármacos deve seguir os princípios das Boas Práticas de Manipulação em Farmácia. A escolha desse tipo de sistema pode garantir a qualidade da assistência à saúde, reduzindo chance de erros ${ }^{(9-10)}$.

A American Society of Hospital Pharmacists (ASHP) recomenda o uso da distribuição por dose unitária e o sistema para preparação de medicação intravenosa realizada pela farmácia para evitar erros na medicação em um hospital(11).

Mas não existem estudos que avaliaram os custos e as consequências da incorporação desta tecnologia na UTIN. Parece oportuna a avaliação da melhor estratégia para a realização da mistura de medicamentos injetáveis nestas unidades, como forma de contribuir para a prevenção e/ou redução de danos relacionadas a infusão de medicamentos.

A questão de pesquisa foi: a central de mistura intravenosa no preparo de medicamentos injetáveis é custo-efetiva para redução de erros quando comparado ao preparo realizada pelo enfermeiro na UTIN? Objetivou-se, no estudo, analisar o custoefetividade da incorporação da central de mistura intravenosa para o preparo de medicamentos injetáveis em unidade UTIN.

\section{MÉTODO}

Avaliação de custo-efetividade, realizada a partir de um modelo matemático utilizando árvore de decisão. Em modelagens matemáticas, o termo cenários é utilizado para se referir a situações hipotéticas, baseadas em pressupostos, que podem ser semelhantes àquelas que eventualmente acontecem no mundo real. Portanto, não foi utilizado nesse estudo nenhum cenário físico.

Os parâmetros imputados no modelo foram baseados em uma coorte hipotética de pacientes e as estimativas de custos e de efetividade, extraídas de estudos primários e secundários. Não houve a necessidade de apreciação do protocolo de pesquisa pelo Comitê de Ética, por se tratar de uma modelagem matemática baseada em dados hipotéticos e pressupostos teóricos. 
A Avaliação Tecnológica em Saúde (ATS) é uma forma abrangente de pesquisar as consequências técnicas, econômicas e sociais, de curto e longo prazo, da utilização das tecnologias em saúde, bem como de seus efeitos diretos e indiretos ${ }^{(12-16)}$.

Modelos são representações da realidade, e, no contexto da análise de custoefetividade, podem ser bastante úteis para informar decisões. São ferramentas analíticas que permitem, a partir de um caso-base, comparar estratégias em saúde. Essa modelagem seguiu as recomendações da Diretriz Metodológica de Avaliação Econômica, do Ministério da Saúde ${ }^{(17)}$.

$\mathrm{Na}$ concepção do modelo, a problemática do estudo tem início com a possibilidade de manipulação de medicações injetáveis em dois diferentes cenários: no cenário de referência, quem faz a manipulação dos medicamentos é o enfermeiro, dentro da UTIN e costuma ser o padrão no Brasil. No alternativo, utiliza-se a Central de Mistura de Injetáveis e a manipulação é feita pelo Farmacêutico, em um local preparado para esse propósito. $O$ caso-base foi composto por esses dois cenários.

No cenário de referência, o mapa de medicamentos é redigido conforme formulário padronizado no setor, variando de hospital para hospital, de uma maneira em geral contendo as seguintes informações: nome da mãe e do recém-nascido, medicamento, dosagem prescrita, via de administração e horário. A incoerência nos dados de identificação do RN e do medicamento interfere na dinâmica de Segurança do Paciente, proposta pelos 9 certos de medicação durante a administração do medicamento no paciente certo ${ }^{(12-13)}$.

Recém-nascidos são mais vulneráveis aos erros de identificação devido à sua inabilidade de participar do processo de identificação, casos de gemelares, semelhança entre bebês nos primeiros dias de vida, similaridade nos números de registro de internação e sobrenomes, além da perda ou remoção de pulseiras de identificação ${ }^{(14-15)}$.

No cenário alternativo, também hipotético, o farmacêutico utiliza equipamentos e softwares para tratar dos mapas e prescrições, atendendo à Resolução da Diretoria Colegiada $n^{\circ}$ 67, de 08 de outubro de 2007 itens de 6.2.1 até 6.2.11, que estabelece, entre outras coisas, que a área física da central de mistura intravenosa deve ser localizada, projetada, construída ou adaptada com uma infraestrutura adequada às atividades a serem desenvolvidas.

A sala destinada ao preparo e envase de preparações estéreis deve ser independente e exclusiva ${ }^{(1-4)}$. A sala destinada à limpeza e higienização dos produtos farmacêuticos, produtos para saúde e materiais de embalagem utilizada no preparo das misturas intravenosas possuem classificação Grau D (100.000 partículas/ pé cúbico ar). Nas áreas de preparo, todas as superfícies devem ser revestidas de material resistente aos agentes sanitizantes, lisas e impermeáveis para evitar acúmulo de partículas e microrganismos, possuindo cantos arredondados ${ }^{(1-3)}$.

Em ambos os cenários, com diferentes probabilidades e com diferentes desfechos, erros e sucesso no preparo dos medicamentos podem ocorrer. No modelo proposto (Figura 1), considerou-se como complicações os eventos adversos medicamentosos, especificamente, erros de dosagem no preparo do medicamento. Portanto, o desfecho de interesse dessa análise foi erro de dose evitado. 


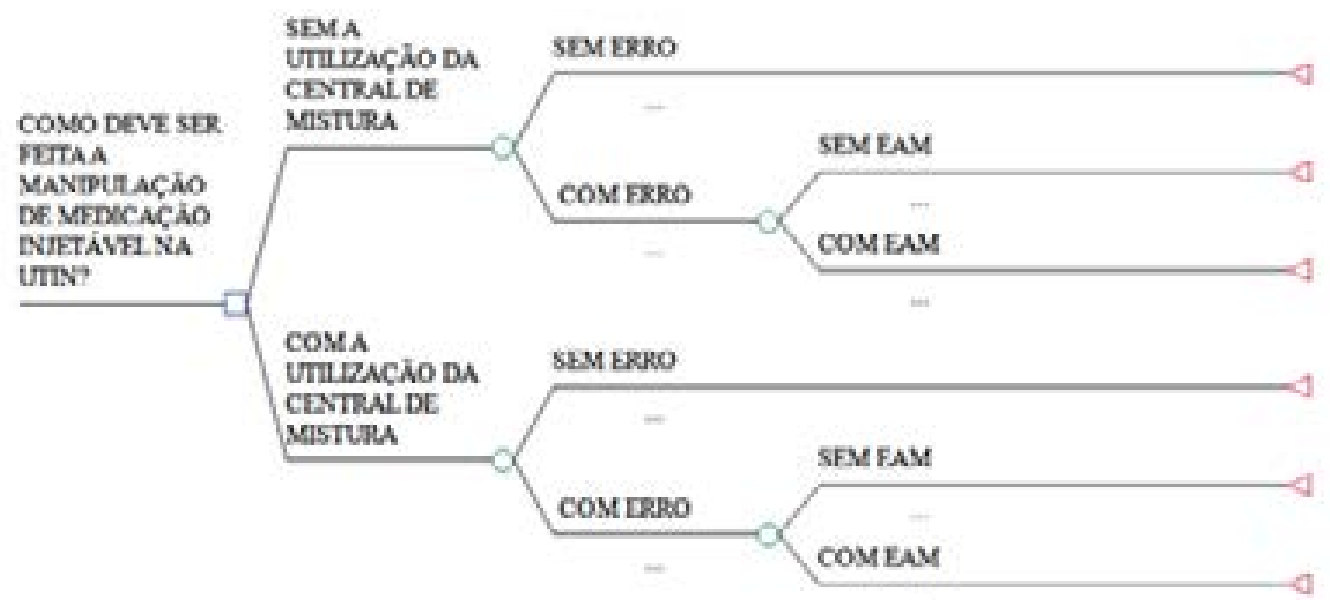

Figura 1 - Estrutura do modelo da árvore de decisão. Rio de Janeiro, RJ, Brasil, 2017 Fonte: Autor, com auxílio do software TreeAge®

Foram utilizados dados primários oriundos dos relatórios gerados pelo EpiMedSolutions e dados secundários obtidos a partir de uma revisão de sistematizada da literatura, para estimar a efetividade, e Banco de Preços em Saúde e Secretaria de Estado de Trabalho e Renda, para estimar os custos.

As incertezas foram tratadas pela análise de sensibilidade probabilística com simulações de Monte Carlo (10.000 simulações), considerando as variáveis de custo, para as quais foram atribuídas distribuições Gama e as variáveis de efetividade probabilidade, para as quais foram atribuídas distribuições Beta.

Os seguintes pressupostos foram considerados no modelo: a perspectiva foi do SUS; o horizonte temporal foi de 1 ano; não foi aplicada taxa de desconto ou de inflação para custo ou efetividade. O tempo médio de internação na UTIN foi estimado em 20 dias ${ }^{(15-18)}$.

Cada prematuro recebeu diariamente de 15 a 20 doses de medicamentos injetáveis (média de 18 medicamentos) ${ }^{(19)}$ e entre 15\% a 69,6\% dos erros de medicamentos ocorrem durante a fase de preparação. A efetividade no cenário de referência foi estimada entre 30,4 e $85 \%$ e no alternativo, entre $63,5 \%$ e $81,1 \%(4,20,23)$.

As taxas de erro de dose e de aumento do tempo de internação após a ocorrência do erro foram respectivamente de 14 a $38 \%$ e $26 \%(2,21,22)$. Na ocorrência de eventos adversos medicamentosos graves, a internação na UTI poderia se prolongar por mais 17 a 28,3 $\operatorname{dias}^{(24-25)}$.

O custo das diárias variou de R\$ 3.002,4 a R\$ 8.893,37 (Secretaria Estadual de Saúde - SES-RJ), e o custo com eventos adversos evitáveis, R\$3.200,00 por dia de internação. Para cada erro que resultou em prolongamento do tempo de internação, mais 17 dias de internação foram acrescentados ao tempo total de internação(26); o custo diário da Central de Misturas Intravenosa foi de R $\$ 475,92$. Esse custo inclui o custo de 24 horas de trabalho de 02 farmacêuticos que farão o preparo de 18 doses de medicamentos injetáveis (média diária de doses realizadas no RN internado na UTIN, encontrado na literatura) e o custo mensal de uso do filtro da câmara de fluxo laminar.

A disposição de pagar (Willingness to Pay - WTP) foi estimada em 01 PIB per capita $\mathrm{R} \$ 28.105,41$, considerando o ano de 2016 (PIB per capita de USD 8.649,95) e a cotação do dólar em 29/12/2016 que foi de $R \$ 3,2492$.

O software TreeAge ${ }^{\circledR} 2011$ Pro Suite (TreeAge software, Willianstown, MA, EU) foi utilizado para a construção do modelo, no formato de árvore de decisão e para a análise de custo-efetividade. 
A Central de Mistura Intravenosa pode ser a melhor alternativa para evitar erros no preparo de medicamentos intravenosos. O gráfico de custo-efetividade (Figura 2) revela que é possível, no caso base, obter uma efetividade média de até 0.96 utilizando a Central de Mistura Intravenosa, a um custo que pode variar de R\$17.400,00 a R\$17.600,00.

\section{Cost-Efrectiveness Analysis}

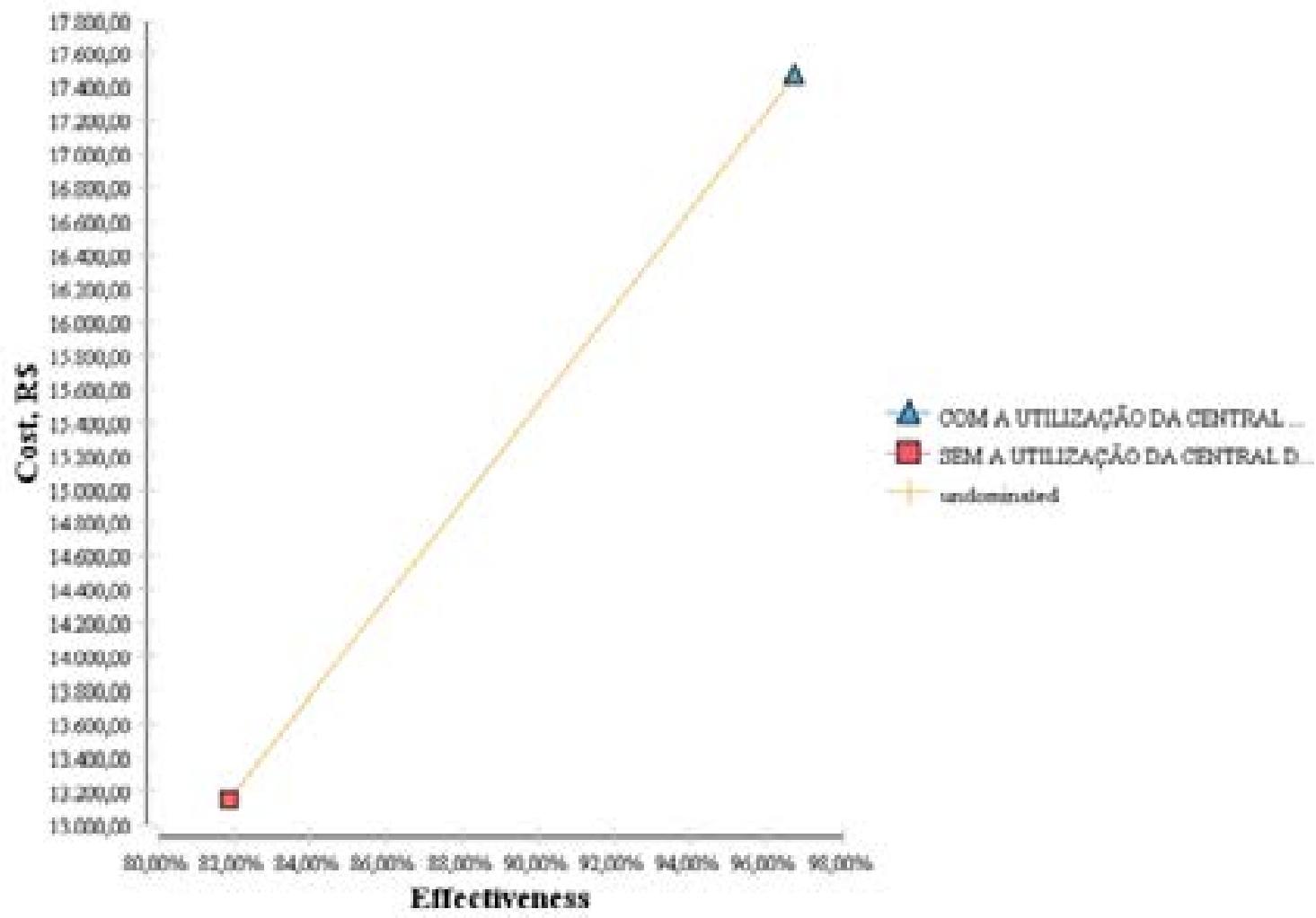

Figura 2 - Gráfico da análise de Custo-Efetividade. Rio de Janeiro, RJ, Brasil, 2017 Fonte: Autor, com auxílio do software TreeAge ${ }^{\circledR}$

Um EAM evitado pode representar uma economia significativa de recursos e vidas salvas, tendo em vista o potencial de morte relacionado a esses eventos. A análise de custoefetividade revelou um ICER de $\mathrm{R} \$ 26.785,61$, um pouco abaixo do limiar de disposição de pagar definido no caso-base.

A curva de aceitabilidade (Figura 3) mostra que a probabilidade da Central de Mistura Intravenosa ser custo-efetiva só é maior a partir de uma disposição de pagar de mais de $\mathrm{R} \$ 87.000,00$, bem acima do limiar de disposição de pagar assumida no modelo ( $\mathrm{R} \$ 28.105,41)$. Com a disposição de pagar assumida no modelo, a probabilidade de ser custo-efetiva é de pouco mais de $35 \%$. 


\section{CE Acceptability Curve}

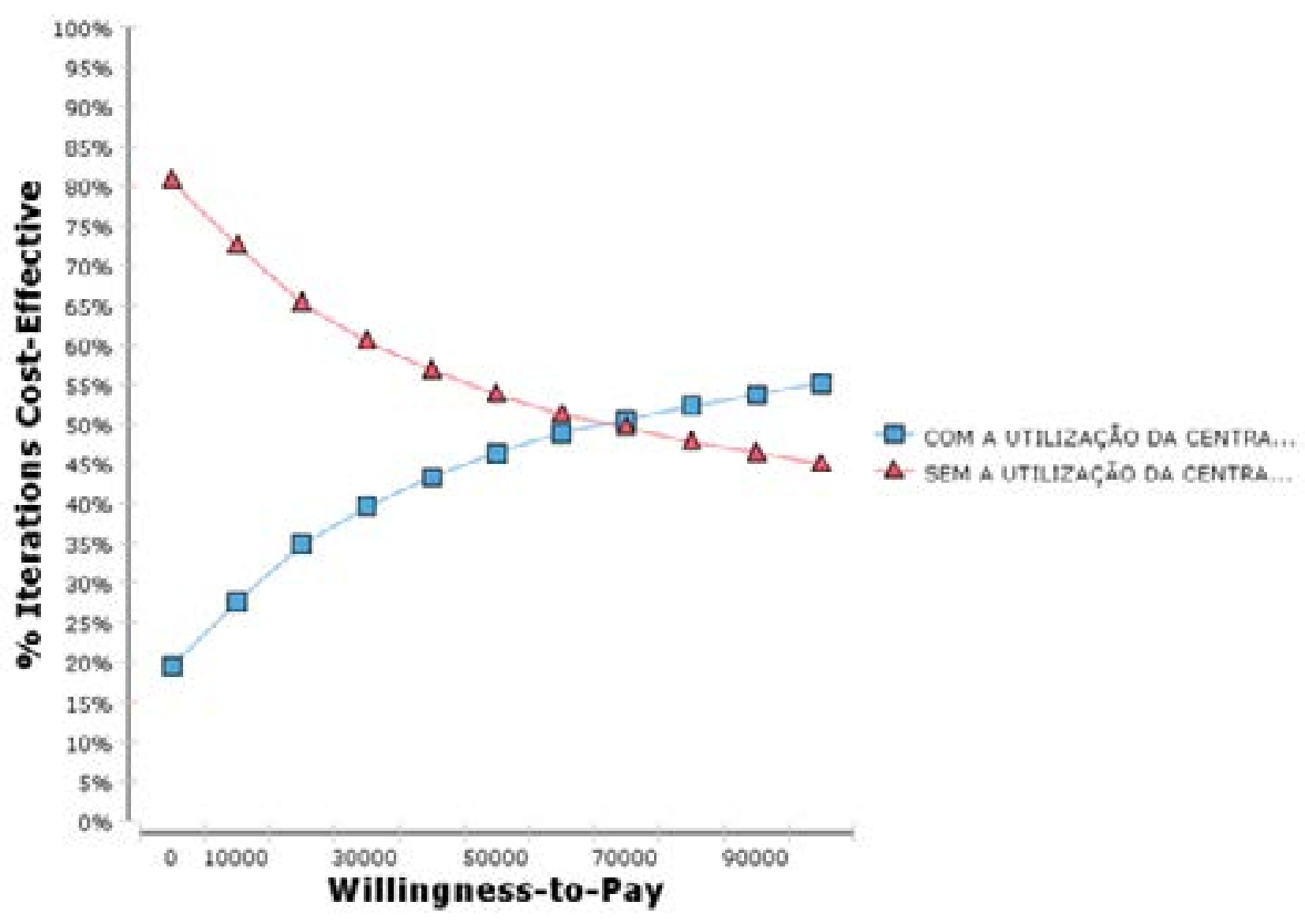

Figura 3 - Gráfico de Curva de Aceitabilidade de Custo-Efetividade. Rio de Janeiro, RJ, Brasil, 2017 Fonte: Autor, com auxílio do software TreeAge $\mathbb{B}$

A análise probabilística realizada a partir de 10.000 simulações de Monte Carlo de segunda ordem para tratar das incertezas relacionadas a variabilidade dos parâmetros imputados no modelo, considerou um intervalo de confiança, representado pela elipse no Scatterplot (Figura 4), de 95\% e o limiar de disposição para pagar, de R\$ 56.000,00 (duas vezes o PIB per capita que seria $\mathrm{R} \$ 28.105,41)$. 


\section{Cost-Efrectiveness Scatterplot}

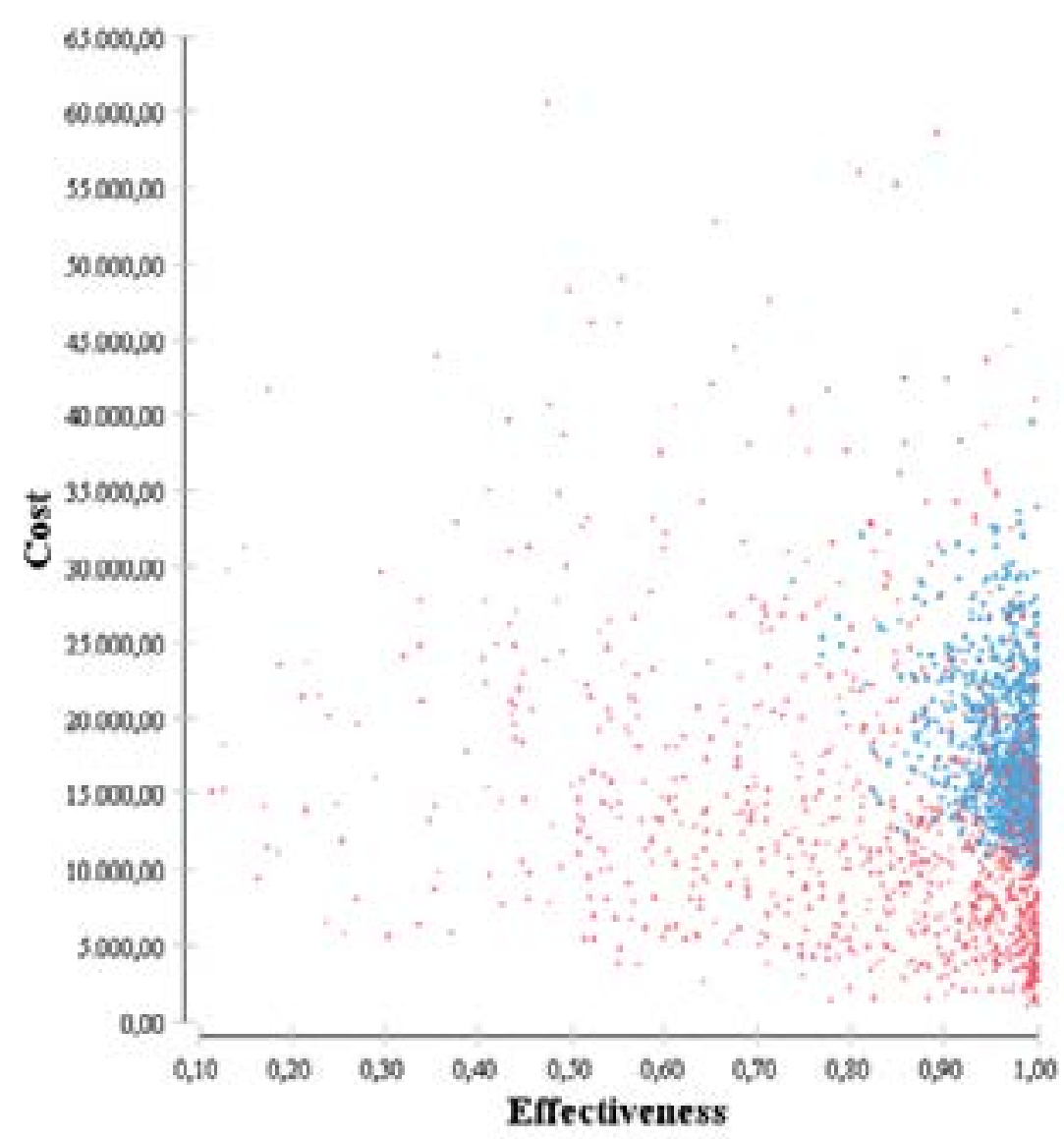

Figura 4 - Scatterplot do Custo-Efetividade. Rio de Janeiro, RJ, Brasil, 2017

Fonte: Autor, com auxílio do software TreeAge ${ }^{\circledR}$

Foi possível constatar no Scatterplot que a efetividade da Central de Mistura Intravenosa variou entre 75 e 95\% (círculo azul no gráfico), com pouca dispersão. Com relação aos custos, a dispersão foi um pouco maior, com maior concentração das simulações entre as faixas de $\mathrm{R} \$ 10.000,00$ a $\mathrm{R} \$ 56.000,00$. Ele revelou ainda uma grande dispersão em relação à efetividade do preparo tradicional realizada pelo Enfermeiro (triângulo vermelho no gráfico), variando entre $10 \%$ e $100 \%$, com maior concentração das simulações na faixa de $50 \%$ a $100 \%$. Em relação aos custos, a dispersão foi bem parecida com a dispersão observada na Central de Mistura Intravenosa, porém com uma variabilidade discretamente maior.

A análise probabilística demonstrou ser muito grande a probabilidade de a Central de Mistura Intravenosa ser realmente mais custo-efetiva que o preparo tradicional realizada pelo Enfermeiro, ratificando o que já havia sido revelado pela curva de aceitabilidade, que demonstrou que a Central de Mistura Intravenosa passa a ter maior probabilidade de ser custo-efetiva comparada ao preparo tradicional realizada pelo Enfermeiro, a partir de um limiar de aproximadamente R\$10.000,00.

\section{DISCUSSÃO}

As avaliações econômicas em saúde são um tipo de estudo que utiliza uma abordagem metodológica e um delineamento pouco convencional e de difícil interpretação por parte daqueles que desconhecem a abordagem. No Brasil, esse tipo de estudo ainda é muito 
incipiente e escasso.

Um dos grandes desafios para o SUS é a implementação de soluções de grande impacto funcional, aliada a um baixo custo operacional, que possam contribuir para a melhoria da qualidade do setor, facilitar o acesso, prover organização e agilidade na assistência à saúde. A resistência à necessidade de alto investimento financeiro, sem considerar os benefícios justificáveis oferecidos, tem mostrado a não ocorrência da implantação da Central de Misturas Intravenosas, um dos requisitos na adoção do sistema de distribuição por dose unitária.

Nessa avaliação, resultados de uma tese de doutorado, desenvolvida por uma enfermeira com expertise na área de neonatologia, foi possível estimar a razão de custoefetividade incremental (ICER) da utilização da Central de Mistura Intravenosa como alternativa custo-efetiva, predita no modelo de árvore de decisão, que deve, portanto, ser incorporada como a melhor opção em termos de tecnologia para evitar erros de dose no preparo de medicamentos intravenosos em UTIN, mas considerando o limiar de disposição de pagar.

A American Society of Health-System Pharmacists (ASHP) cita a distribuição por dose unitária e o sistema para preparação de medicamentos intravenosa realizada pela farmácia como recomendações importantes para evitar erros de medicamentos em um hospital (11).

Os resultados desse estudo devem servir de alerta para que os enfermeiro e os gestores dos serviços de saúde repensem os sistemas tradicionais de preparo de medicamentos intravenosos, pois se questiona sobre o preparo dos medicamentos injetáveis realizado pela equipe de enfermagem, tanto do ponto de vista técnico quanto do ponto de vista legal, considerando a Lei do Exercício Profissional dos Profissionais de Enfermagem, os espaços físicos destinados à manipulação e ao preparo, e a habilidade técnica e conhecimento que podem levar ao uso incorreto do material, comprometendo a segurança, eficácia, efetividade e eficiência do preparo das doses desses medicamentos.

A existência da Central de Misturas Intravenosas exige maior interação da equipe multidisciplinar na unidade, pois o farmacêutico debate com a equipe médica o medicamento prescrito e posteriormente com a equipe de enfermagem, o medicamento já preparado, proporcionando uma melhoria da qualidade da assistência prestada ao paciente ${ }^{(27)}$.

De ordem econômica, a centralização do preparo de medicamentos injetáveis influencia na redução do custo, principalmente com a utilização racional dos medicamentos. Estudos realizados pelo Instituto da Criança mostraram que, com a disposição de medicamentos unitarizados, a farmácia pode reduzir o consumo interno em até $35 \%$.

Os gastos relacionados à implantação da Central de Mistura Intravenosa, em relação aos custos totais do hospital, podem representar um valor em torno de $5 \%$ a $20 \%$ com crescimento médio de $25 \%$ ao ano. Portanto, torna-se de extrema importância a existência de sistemas de distribuição de medicamentos que racionalizem este processo ${ }^{(28)}$.

Na prática, a implantação da Central de Mistura Intravenosa pode impactar diretamente na equipe de enfermagem. No cenário tradicional, onde o enfermeiro prepara o medicamento dentro da UTIN, o ambiente extremamente agitado pela complexidade da assistência pode acarretar inconformidade medicamentosa. Nessa situação, faz-se necessário propiciar um ambiente seguro para o preparo de medicamentos. A Central de Misturas Intravenosa pode reduzir de 8 para 2 horas o tempo gasto pelo enfermeiro para a administração de medicamentos injetáveis, proporcionando disponibilidade de tempo a esses profissionais para realização de suas atividades de forma mais segura ${ }^{(4)}$.

Para os clientes, neste caso RN na grande maioria prematura, a implantação da Central de Mistura Intravenosa representa maior segurança e qualidade no tratamento. Para a instituição, a proporção de substancial economia, e para o profissional de enfermagem, a ampliação de conhecimentos, maior integração a equipe com serviço mais seguro e mais tempo para estar à beira do leito do paciente. Especificamente na Farmácia, essa 
implantação permite o retorno da atividade de preparo de medicamentos, que é de fato sua responsabilidade.

Como qualquer análise econômica desenvolvida a partir de modelagens matemáticas, incertezas de ordem estrutural, analítica e paramétricas, presentes no modelo, podem impor limitações, sobretudo no que se refere à validade externa e poder de extrapolação dos seus resultados.

Como os modelos são representações da realidade e, como tal, são criados na tentativa simular cenários que se aproximem o máximo possível do mundo real, é preciso admitir que uma das principais limitações desse e de qualquer outra modelagem para análise econômica em saúde é a dificuldade de interpretação dos seus resultados pelos gestores e profissionais de saúde que querem informar decisõ̉es a partir dos seus resultados. A dificuldade na interpretação do ICER é um bom exemplo.

Nessa avaliação econômica, o modelo prediz que o custo para se evitar adicionalmente um erro de dose no preparo de medicamentos injetáveis (ICER), é de R\$26.785,61, utilizando a central de mistura de injetáveis. A princípio esse custo parece realmente alto, mas é preciso considerar que, no modelo avaliado, o custo total do cenário alternativo (cenário que utiliza a central de mistura de injetáveis) incluiu o custo com a aquisição de equipamentos e realização de obras necessárias no espaço físico, para implantação da central.

A análise do impacto orçamentário, o que não foi possível ser feito por conta do tempo disponível para a conclusão da tese, poderia facilitar e melhor orientar a interpretação dos resultados dessa avaliação. A impossibilidade de realização do impacto orçamentário, deve, portanto, ser considerada uma limitação do estudo. Por isso, é importante destacar que qualquer tentativa de extrapolar os resultados dessa avaliação para cenários que possam ser muito diferentes dos pressupostos assumidos no modelo, precisa ser feita com muita cautela.

As análises econômicas em saúde e seus diferentes tipos de estudos e de abordagens, tem sido, para a área de enfermagem, uma oportunidade ímpar para ampliar o escopo de abordagens metodológicas na área. Convém ressaltar as dificuldades encontradas para estimar a efetividade dos cenários analisados no estudo, diante da escassez dos estudos abordando essa temática, sobretudo, desenvolvidos no Brasil.

\section{CONCLUSÃO}

A razão de custo-efetividade incremental (ICER) foi de $\mathrm{R} \$ 26.785,61$, o que nos permite inferir que, no caso-base, o uso da central de mistura intravenosa se mostrou custo-efetivo, considerando a disposição de pagar.

A extrapolação dos resultados dessa análise deve ser feita com cautela, diante das incertezas estruturais e paramétricas do modelo que não foram tratadas na análise de sensibilidade. Em relação à validade interna dos resultados de estudos utilizados como pressupostos no modelo, considerando uma escala de valoração da confiabilidade das evidências científicas, muito embora o valor dos estudos observacionais e revisões possam ser reduzidos, na ausência de dados mais consistentes, e para fins de avaliação de efetividade, sua contribuição foi bastante útil no modelo.

Esta análise econômica pode representar uma nova perspectiva para a abordagem da problemática dos EAM relacionados ao preparo de medicamentos intravenosos nas UTIN, quase sempre abordada na literatura científica a partir de dois enfoques quase sempre dissociados um do outro. O primeiro, baseado na preocupação com o crescente aumento das taxas de EAM nessas unidades, o que tem concorrido para o avanço do conhecimento científico acerca da melhor estratégia para reduzi-los, e o segundo relacionado ao crescente aumento dos custos hospitalares associados à incorporaçã̃o de tecnologias e ao próprio 
EA, tornando-se explícito no crescente número de estudos publicados sobre o tema, ainda que seus resultados não tenham força de evidência para oferecer suporte sólido para condutas sobre essa situação, e no Brasil, eles ainda são incipientes.

Ao se evitar EAM, proporcionam-se ganhos e benefícios para a população estudada, e o tamanho desse benefício é diretamente proporcional ao tamanho da efetividade. O estudo demonstrou que, ao utilizar a Central de Mistura Intravenosa, o custo-efetividade é maior quando comparado com o cenário aonde o enfermeiro prepara os medicamentos intravenosos no espaço da UTIN.

Como complemento necessário ao estudo para que possa informar decisão, sugerese que sejam realizadas análises de impacto orçamentário da adoção da estratégia mais custo-efetiva de acordo com a população-alvo e a realidade institucional, o que não foi possível nessa análise econômica.

O estudo pode contribuir para reduzir incertezas na tomada de decisões acerca da incorporação de tecnologias para redução de erros de dose na administração de medicamentos em UTI pediátrica e neonatal.

\section{REFERÊNCIAS}

1. Belela ASC, Pedreira MLG, Peterlini MAS. Erros de medicação em pediatria. Rev. bras. enferm. [Internet]. 2011 [acesso em 01 maio 2019]; 64(3). Disponível em: http://dx.doi.org/10.1590/S003471672011000300022 .

2. Lanzillotti LS, Seta MH de, Andrade CLT de, Mendes JWV. Eventos adversos e outros incidentes em unidades de terapia intensiva neonatal. Cienc. saude colet. [Internet]. 2015 [acesso em 01 maio 2019]; 20(3). Disponível em: http://dx.doi.org/10.1590/1413 81232015203.16912013.

3. Schatkoski AM, Wegner W, Algeri S, Pedro ENR. Segurança e proteção à criança hospitalizada: revisão de literatura. Rev. Latino-Am. Enfermagem [Internet]. 2009 [acesso em 01 maio 2019]; 17(3). Disponível em: http://dx.doi.org/10.1590/S0104-11692009000300020.

4. Koumpagioti D, Varounis C, Kletsiou E, Nteli C, Matziou V. Evaluation of the medication process in pediatric patients: a meta-analysis. J. Pediatr. [Internet]. 2014 [acesso em 01 maio 19]; 90(4). Disponível em: http://dx.doi.org/10.1016/j.jped.2014.01.008.

5. Davies MJ, Kotadia AMH, Hannan A, Alqarni $H$. The attitudes of pharmacists, students and the general public on mHealth applications for medication adherence. J. Pharm. Pract [Internet]. 2015 [acesso em 01 maio 2019]; 13(4). Disponível em: http://dx.doi.org/10.18549/PharmPract.2015.04.644.

6. Costa EA, Araújo PS, Pereira MT, Souto AC, Souza GS, Guerra JAA, et al. Technical issues and conservation conditions of medicines in the primary health care of the Brazilian Unified Health System. Rev. Saúde Públ. [Internet]. 2017 [acesso em 01 maio 19]; 51(Suppl 2): 12. Disponível em: http://dx.doi. org/10.11606/s1518-8787.2017051007106.

7. Veja EN, Díaz AMA, Gorgas-Torner MQ, Barrios CE, Rubia Nieto A de la. Quality indicators for technologies applied to the hospital pharmacy. Farm Hosp. [Internet]. 2017 [acesso em 01 maio 19]; 41(4). Disponível em: http://dx.doi.org/10.7399/fh.2017.41.4.10698.

8. Barbosa MT de SR, Alves VH, Rodrigues DP, Branco MBLR, Souza R de MP de, Bonazzi VCAM. Quality indicators in support of intravenous therapy in a university hospital: a contribution of nursing. Rev. Pesqui. [Internet]. 2015 [acesso em 01 maio 2019]; 7(2). Disponível em: http://dx.doi.org/10.9789/21755361.2015.v7i2.2277-2286.

9. Machado-Alba JE, Moncada JC, Moreno-Gutiérrez PA. Medication errors in outpatient care in Colombia, 2005-2013. biomed. [Internet]. 2016 [acesso em 01 maio 2019]; 36(2). Disponível em: http:// dx.doi.org/10.7705/biomedica.v36i2.2693. 
10. Silva MJS da, Magarinos-Torres R, Oliveira MA, Osorio-de-Castro CGS. Avaliação dos serviços de farmácia dos hospitais estaduais do Rio de Janeiro, Brasil. Ciênc. saúde coletiva [Internet]. 2013 [acesso em 01 maio 2019];18(12). Disponível em: http://dx.doi.org/10.1590/S1413-81232013001200017.

11. Coyoc RO, Pérez-Reynaud AG, Coello-Reyes LA. Beneficios económicos del uso de un sistema de dispensación em dosis unitárias em hospitales del Instituto Mexicano del Seguro Social. Salud pública Méx [Internet]. 2014 [acesso em 01 maio 2019]; 56(3). Disponível em: http://www.scielo.org.mx/scielo. php?script=sci abstract\&pid=S003636342014000300013\&lng=pt\&nrm=iso.

12. Vásquez AR, Montoya JIE. Apreciaciones de trabajadores de la salud sobre la implementación del sistema de seguridade em la utilización de medicamentos. Rev Univ. salud. [Internet]. 2017 [acesso em 01 maio 2019];19 (1). Disponível em: http://dx.doi.org/10.22267/rus.171901.64.

13. Brasil. Ministério da Saúde. Secretaria de Ciência, Tecnologia e Insumos Estratégicos. Departamento de Ciência e Tecnologia. Diretrizes metodológicas: elaboração de estudos para avaliação de equipamentos médicos assistenciais. Brasília: MS, 2013. [acesso em 10 dez 2017]. Disponível em: http:// www.saude.gov.br/bvs.

14. Truter A, Schellack N, Meyer J C. Identifying medication errors in the neonatal intensive care unit and paediatric wards using a medication error checklist at a tertiary academic hospital in Gauteng, South Africa. S. Afr. j. child health [Internet]. 2017 [acesso em 01 maio 2019]; 11(1). Disponível em: http://dx.doi. org/10.7196/sajch.2017.v11i1.1101.

15. Machado APC, Tomich CSF, Osme SF, Ferreira DM de LM, Mendonça MAO, Pinto RMC, et al. Prescribing errors in a Brazilian neonatal intensive care unit. Cad. saúde Pública [Internet]. 2015 [acesso em 01 maio 2019]; 31(12). Disponível em: http://dx.doi.org/10.1590/0102-311X00194714.

16. Brasil. Ministério da Saúde. Secretaria de Ciência, Tecnologia e Insumos Estratégicos. Departamento de Ciência e Tecnologia. Diretrizes Metodológicas: estudos de avaliação econômica de tecnologias em saúde. Brasília: Ministério da Saúde; 2009. 145p: il. (Série A. Normas e Manuais Técnicos).

17. Brasil. Ministério da Saúde. Secretaria de Ciência, Tecnologia e Insumos Estratégicos. Departamento de Ciência e Tecnologia. Diretrizes metodológicas: elaboração de pareceres técnico-científicos. 4. ed. Brasília: Ministério da Saúde; 2014.

18. Sposito NPB, Rossato LM, Bueno M, Kimura AF, Costa T, Guedes DMB. Avaliação e manejo da dor em recém-nascidos internados em Unidade de Terapia Intensiva Neonatal: estudo transversal. Rev. LatinoAm. Enfermagem [Internet]. 2017 [acesso em 01 maio 2019]; 25(1). Disponível em: http://www.redalyc. org/articulo.oa?id=281449566067.

19. Cadavid JM, Amariles P. Incompatibilidad de medicamentos intravenosos: revisión estructurada. CES Med. [Internet]. 2017 [acesso em 01 maio 2019]; 31(1). Disponível em: http://dx.doi.org/10.21615/ cesmedicina.31.1.6.

20. Volpe CRG, Melo EMM, Aguiar LB de, Pinho DLM, Stival MM. Risk factors for medication errors in the electronic and manual prescription. Rev. Latino-Am. Enfermagem. [Internet]. 2016 [acesso em 01 maio 2019]; 24(2). Disponível em: http://www.scielo.br/pdf/rlae/v24/pt 0104-1169-rlae-24-02742.pdf.

21. Achury Saldanã D, Rodríguez SM, Díaz JC, Cavallo E, Zarate Grajales R, Vargas Tolosa R, et al. Estudio de eventos adversos, factores y periodicidaden pacientes hospitalizados en unidades de cuidado intensivo. Enferm. glob. [Internet]. 2016 [acesso em 01 maio 2019]; 15(42). Disponível em: http://scielo. isciii.es/scielo.php?script=sci arttext\&pid=S1695-61412016000200011\&lng=es.

22. Silva GD da, Silvino ZR, Almeida VS de, Querido DL, Dias PSC, Christovam B y P. Errores de medicaciónen Unidades de Terapia Intensiva Neonatal. Enferm. glob. [Internet]. 2014 [acesso em 01 maio 2019]; 13(33). Disponível em: http://scielo.isciii.es/scielo.php?script=sci arttext\&pid=S169561412014000100019\&lng=es.

23. Ribeiro E. Dose Unitária: Sistema de Distribuição de Medicamentos em Hospitais. Rev. adm. empres. [Internet]. 1993 [acesso em 01 maio 2019]; 33(6). Disponível em: http://dx.doi.org/10.1590/S003475901993000600007. 
24. Ventura CMU, Alves JGB, Meneses J do A. Eventos adversos em Unidade de Terapia Intensiva Neonatal. Rev. bras. enferm. [Internet]. 2012 [acesso em 01 maio 2019]; 65(1). Disponível em: http:// dx.doi.org/10.1590/S0034-71672012000100007.

25. Junkes MB, Pessoa VF. Gasto financeiro ocasionado pelos atestados médicos de profissionais da saúde em hospitais públicos no Estado de Rondônia, Brasil. Rev. Latino-Am. Enfermagem [Internet]. 2010 [acesso em 01 maio 2019]; 18(3). Disponível em: http://www.redalyc.org/articulo.oa?id=281421933016.

26. Bohomol E. Erros de medicação: estudo descritivo das classes dos medicamentos e medicamentos de alta vigilância. Esc. Anna Nery [Internet]. 2014 [acesso em 01 maio 2019];18(2). Disponível em: http:// www.redalyc.org/articulo.oa?id=127730686019.

27. Toro AM. Dispensación de medicamentos em las grandes farmacias de chile: análisis ético sobre laprofesióndel químico farmacéutico. Acta bioeth [Internet]. 2017 [acesso em 01 maio 2019]; 23(2). Disponível em: http://www.redalyc.org/articulo.oa?id=55453395014.

28. Reis CCC dos, Moro MF, Flores S de A, Yui KP, Weise AD. Procedimento de registro dos parâmetros intervenientes na logística reversa em uma farmácia hospitalar. Exacta [Internet]. 20172014 [acesso em 01 maio 2019]; 15(3). Disponível em: http://www.redalyc.org/articulo.oa?id=81052980002.

Recebido: 07/08/2018

Finalizado: 12/08/2019

Autor Correspondente:

Carlos Roberto Lyra da Silva

Universidade Federal do Estado do Rio de Janeiro

Av. Pasteur, 296 - 22290-240 - Rio de Janeiro, RJ, Brasil

E-mail: profunirio@gmail.com

Contribuição dos autores:

Contribuições substanciais para a concepção ou desenho do estudo; ou a aquisição, análise ou interpretação de dados do estudo - LFM, CBM, RCLS, AAFP, VS

Aprovação da versão final do estudo a ser publicado - RCLS, CRLS, VS 